\title{
The Association between Human Leukocyte Antigen Class II DR3-DQ2 Haplotype and Type 1 Diabetes in Children of the East Azerbaijan State of Iran
}

\author{
Sima Mansoori Derakhshan ${ }^{1,2}$; Fatemeh Zeinali Sehrig ${ }^{3}$; Nasrin Sohrabi ${ }^{1,2}$; Siamak Shiva ${ }^{4}$; \\ Behzad Baradaran ${ }^{1}$; Mahmoud Shekari Khaniani ${ }^{1,2, *}$ \\ ${ }^{1}$ Immunology Research Center, Tabriz University of Medical Sciences, Tabriz, IR Iran \\ ${ }_{3}^{2}$ Department of Medical Genetic, Faculty of Medicine, Tabriz University of Medical Sciences, Tabriz, IR Iran \\ ${ }^{3}$ Department of Biological Science, Ahar Branch, Islamic Azad University, Ahar, IR Iran \\ ${ }^{4}$ Department of Pediatrics, Faculty of Medicine, Tabriz University of Medical Sciences, Tabriz, IR Iran \\ *Corresponding Author: Mahmoud Shekari Khaniani, Department of Medical Genetic, Faculty of Medicine, Tabriz University of Medical Sciences, Tabriz, IR Iran. Tel: +98-4113371587, \\ Fax:+98-4113371587, E-mail: Mahmoud.khaniani@gmail.com
}

Received: March 11, 2015; Revised: June 12, 2015; Accepted: July 13, 2015

\begin{abstract}
Background: Type 1 diabetes mellitus (T1D) is an autoimmune disease. Several associations between human leukocyte antigen (HLA) complex and T1D were found in various populations. Associations with various HLA types depend on the investigated populations. However, such associations have not yet been investigated in the East Azerbaijan state of Iran with Turkish ethnicity.

Objectives: The aims of the current study was to describe T1D genetic susceptibility conferred by HLA class II alleles (DRB1 ${ }^{*} 0301$, DQA1*0501 and DQB1*0201) and to determine haplotype frequencies among T1D patients.

Patients and Methods: This study was a case-control study. The number of samples was determined using the Cochran formula. Eighty unrelated T1D patients, including 42 (52.5\%) females and 38 (47.5\%) males, were randomly recruited from the East Azerbaijan state of Iran. Typing of HLA was performed by polymerase chain reaction-sequence-specific priming (PCR-SSP) on DNA extracted from peripheral blood mononuclear cells of 80 unrelated patients and 80 unrelated healthy control donors, who were selected randomly. For haplotype analysis, the logistic regression model was performed that allows joint estimation of Single-nucleotide polymorphisms(SNPs) via haplotypes.

Results: The frequency of drb1*0301 (82.5\% vs. $11.3 \%)$, dqa1*0501 ( $82.5 \%$ vs. $36.3 \%)$ and dqb1*0201 (81.3\% vs. 35\%) were significantly higher among patients compared with that of healthy subjects.

Conclusions: Our investigation demonstrated that there is a highly significant association between the studied alleles and T1D. It can be construed that haplotype HLA-DR3-DQ2 has a very modest effect with respect to the risk of T1D.
\end{abstract}

Keywords: Haplotype; Genotype; HLA-DRB1; HLA-DQB1

\section{Background}

Type 1 diabetes (T1D) is an organ-specific, autoimmune, common chronic disease of children and young adults $(1,2)$. It results from an autoimmune CD4+ and CD8 + Tcell-directed destruction of insulin-producing pancreatic islet $\beta$ cells, causing irreversible hyperglycemia and various other complications. This autoimmune response involves both the cellular and humoral arms of the immune system; however, cell-mediated processes prevail. The initiating event remains unknown (3-5). It is a genetically determined disease as proved by family studies on disease concordance among siblings. The probability to have the disorder appeared to be approximately $40 \%$ for monozygotic and 5\% for dizygotic siblings. However, if the latter are HLA-identical, this number increases to $20 \%$ (1).

Type 1 Diabetes is a polygenic disease that develops due to interaction between environmental and genetic fac- tors $(6,7)$. About eighteen regions of the genome, mapped to the loci IDDM1 to IDDM18, have been linked with the risk of T1D; of these, the human leukocyte antigen (HLA) region (identified as IDDM1) accounts for more than half of the genetic susceptibility to T1D $(1,8-10)$. These facts indicate a substantial role for HLA in the immunopathogenic processes leading to disease development. Differences in HLA genes have been found to be genetic risk factors for T1D; yet, they themselves do not cause the disease when other key genes are involved $(11,12)$. Over the past 40 years, research investigations have focused not only on the determination of alleles of the HLA genes, which account for the association with T1D, but also on other genetic loci, in addition to HLA, which contribute to the risk of T1D; dozens of such loci have been discovered to show associations with T1D $(13,14)$. 
Human leukocyte antigen has an important role in self/ non-self-recognition, and includes highly polymorphic class I (HLA-A, -B and -C), class II (HLA-DP, -DQ and -DR) and class III loci, each of which are subdivided to distinct regions $(12,15)$. Genetic mapping of these regions in HLA class II shows that the most important genes are DQB1 and DRB1. Alleles of the DQB1 gene are often associated with alleles of the DRB1 gene, and variants of both or either gene may confer an increased risk of diabetes (16). More than 315 alleles of HLA-DRB1 have been discovered, constituting the most polymorphic protein-encoding region of the human genome $(17,18)$. The contribution of specific HLA haplotypes toward T1D susceptibility depends on the ethnic/racial background $(10,19)$. This indicates that the association of a specific class II allele and DRB1-DQB1 haplotype with T1D risk must be evaluated in the context of specific ethnic/ racial backgrounds $(19,20)$. According to the above points, the current study was the first molecular report on the association of the DR3-DQ2 haplotype of HLA class II with T1D in East Azerbaijan state of Iran.

\section{Objectives}

Comparative analysis of the HLA class II DR and DQ alleles and haplotypes has revealed differences in the distribution of these alleles among different ethnic groups and has reflected ancient and recent admixtures between different populations (21). This study investigated the in fluence of DR3-DQ2 haplotype on T1D with complete molecular genotyping: the allele and haplotype frequencies of HLA-DRB1*0301, DQA1*0501 and DQB1*0201 are presented, which can also be useful as basic data for anthropology and disease association studies.

\section{Patients and Methods}

\subsection{Subjects}

This study was a case-control study. The number of samples was determined using the following Cochran formula (Equation 1):

$$
n=\frac{z^{2} p(1-p)}{d^{2}}=\frac{1.96^{2} \times 0.5 \times 0.5}{0.11^{2}}=80
$$

Eighty unrelated T1D patients, including 42 (52.5\%) females and 38 (47.5\%) males, were randomly recruited from the East Azerbaijan state of Iran. In addition, 80 control subjects with normal fasting/random blood glucose levels and no family history of T1D or other autoimmune diseases were included in this study. To compare the two independent groups, t-test was used due to the normality of the data. Because of the presence of more than one observer, kappa coefficient was also calculated (Table 1). It should be noted that all obtained data by this study were analyzed by the Epidemiology and Biostatistics Center of Tabriz University of Medical Sciences.

Type 1 Diabetes was diagnosed by a consultant endocrinologist according to glycemic criteria of the American diabetes association (ADA) guidelines (22), i.e. elevated fasting glucose levels ( $\geq 7.0 \mathrm{mmol} / \mathrm{L}(126 \mathrm{mg} / \mathrm{dL}$ ) and twohour plasma glucose $\geq 11.1 \mathrm{mmol} / \mathrm{L}(200 \mathrm{mg} / \mathrm{dL}))$. Glucose measurement was carried out on the Roche/Hitachi 911 device. For avoiding instrumental error, all equipment were calibrated before use. Table 1 shows the characteristic of T1D patients and normal subjects.

\begin{tabular}{|c|c|c|c|}
\hline Total Numbers & Patients $(n=80)$ & $\operatorname{Control}(\mathbf{n}=\mathbf{8 0})$ & $\begin{array}{l}\text { (For Comparison Between } \\
\text { Control and Patient Groups) }\end{array}$ \\
\hline \multicolumn{4}{|l|}{ Gender } \\
\hline Female & $42(52.5 \%)$ & $41(51.25 \%)$ & 0.44 \\
\hline Male & $38(47.5 \%)$ & $39(48.75 \%)$ & \\
\hline Mean age, $y$ & $13.33 \pm 1.37$ & $11.17 \pm 2.65$ & 0.81 \\
\hline Family history of T1D & - & - & \\
\hline FBS, $\mathrm{mg} / \mathrm{dL}$ & $130 \pm 4$ & $98 \pm 2$ & $0.043^{b}$ \\
\hline LDL-C & 143 & 98 & $0.039^{b}$ \\
\hline HDL-C & 42 & 53 & $0.048^{b}$ \\
\hline TG & 232 & 127 & $0.012^{b}$ \\
\hline Total cholesterol & 203 & 155 & $0.032^{b}$ \\
\hline $\mathrm{Cr}$ & 0.9 & 0.5 & 0.067 \\
\hline HbA1C & $6.50 \%$ & $5 \%$ & 0.07 \\
\hline
\end{tabular}

a Abbreviations: Cr, Creatinine; FBS, Fasting blood sugar; HDL, High-Density Lipoprotein; LDL, Low-Density Lipoprotein; TG, Triglyceride.

$\mathrm{b}$ The difference was significant at the 0.05 level. 
Mansoori Derakhshan S et al.

\subsection{Ethics}

This study was approved by the regional committee of ethics of the Tabriz university of medical sciences by issue number 2.2-2/92 on the $16^{\text {th }}$ of August 2013. Written informed consent was obtained from the parents of all the children who participated in this study. All of the authors of this research confirmed that the patients' privacy was protected and the entire process was done with a prior written consent.

\subsection{Human Leukocyte Antigen Genotyping}

Total genomic DNA was extracted from the peripheral blood cells of study participants using the salting-out method. Polymerase Chain Reaction-Sequence-Specific Priming (PCR-SSP) technique by a thermocycler (Eppendrof) was used for genotyping of DRB1*0301, DQA1*0501, and DQB1*0201 haplotypes, as described (23). Sequences of primers (Macrogen) are shown in Table 2. The PCR products were analyzed by agarose gel electrophoresis (PEQlab) using 1\% (w/v) agarose (Cinnagen) gel, and visualized by staining with the simply blue safe stain (Fermentas).

\subsection{Data Analysis}

For haplotype analysis, the logistic regression model was used. Data were analyzed using the STATA11 statistical software package (STATA Corporation, Texas). For preliminary descriptive statistics, variant analysis was done using chi-square test and calculation of odd ratios (ORs) with 95\% confidence interval (CI). The calculated ORs presented the likelihood of developing T1D for subjects with minor alleles compared to major alleles. $\mathrm{P}$ values were also reported. P values of $<0.05$ were considered statistically significant. All the tests involved a tailed analysis.

\section{Results}

\subsection{Association of the Alleles drb1*0301, DQA1*0501 and DQB1 ${ }^{*} 0201$ with Type 1 Diabetes}

Allele frequencies of HLA-DRB1*0301, DQA1*0501 and DQB1*0201 in T1D patients and healthy controls are shown in Table 2. The DRB1*0301(82.5\% vs. 11.3\%), DQA1*0501(82.5\% vs. $36.3 \%$ ) and DQB1*0201 (81.3\% vs. 35\%) alleles had higher frequencies among patients compared to controls. Figure 1 displays a representative gel picture of DQA1*0501 PCRSSP result.4.2. Association of the Haplotype DRB1*0301DQA1*0501 with Type 1 Diabetes

The association of DRB1*0301-DQA1*0501 haplotype with T1D was investigated, and four haplotype frequencies were calculated. The most frequent haplotype, $\mathrm{DRB1}^{*} 0301{ }^{+}$-DQB1 ${ }^{*} 0302{ }^{+}(\mathrm{OR}=0.78,95 \% \mathrm{CI}=243.01 \pm$ 453), was used as a reference haplotype for the calculation of ORs. All information about the various haplotypes is shown in Table 3.

\subsection{Association of the Haplotype DRB1*0301- $D Q A 1^{*} 0501-D Q B 1^{*} 0201$ with Type 1 Diabetes}

The association of the DRB1*0301-DQA1*0501-DQB1*0201 haplotype with T1D was investigated, and four haplotype frequencies, the haplotype associations and related ORs were calculated (Table 4 ). The most frequent haplotype $\left(\mathrm{DRB1}{ }^{*} 0401^{+}{ }^{-\mathrm{DQB} 1}{ }^{*} 0302^{+}{ }^{-D Q A 1}{ }^{*} 0301^{+}\right.$) was used as a reference haplotype for the calculation of ORs.

Table 2. Sequences of Primers and Human Leukocyte Antigen Allele Distribution in Subjects with Type 1 Diabetes and Control a,b

\begin{tabular}{|c|c|c|c|c|c|c|}
\hline Sequences of Primers & Product Size & T1D & Control & OR & Kappa & 95\% CI \\
\hline \multicolumn{7}{|l|}{ DRB1*0301 } \\
\hline F: TACTTCCATAACCAGGAGGAGA & \multirow{2}{*}{$151 \mathrm{bp}$} & $+: 66(82.5)$ & $+: 9(11.3)$ & 37.2 & 0.189 & $15.1-91.7$ \\
\hline R: TGCAGTAGTTGTCCACCCG & & - : :14 (17.5) & - :71(88.8) & & & \\
\hline \multicolumn{7}{|l|}{ DQA1*0501 } \\
\hline F: ACGGTCCCTCTGGCCAGTA & \multirow{3}{*}{$186 \mathrm{bp}$} & $+: 66(82.5)$ & $+: 29(36.3)$ & 8.3 & 0.181 & $4.0-17.3$ \\
\hline R:AGTTGGAGCGTTTAATCAGAC & & -::14 (17.5) & $-: 51(63.8)$ & & & \\
\hline \multicolumn{6}{|l|}{ DQB1*0201 } & \\
\hline F: GTGCGTCTTGTGAGCAGAAG & \multirow{2}{*}{205 bp } & $+: 65(81.3)$ & $+: 28(35)$ & 8.1 & 0.19 & $3.9-16.6$ \\
\hline R: GCAAGGTCGTGCGGAGCT & & - :15 (18.8) & - :52 (65) & & & \\
\hline
\end{tabular}

\footnotetext{
a Abbreviations: CI, Confidence interval; OR, Odds Ratio.

b P Value $=0.0001$
} 
Figure 1. DQA1*05 Gel Image

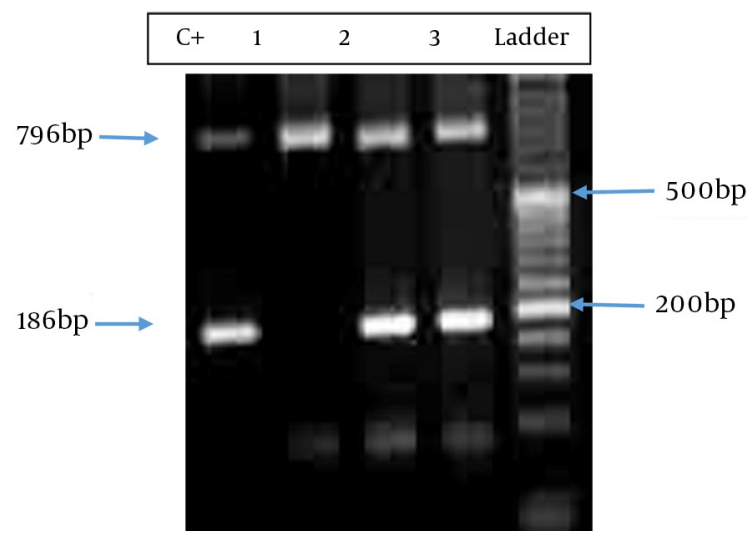

$\mathrm{C}+$ was the positive control. $\mathrm{C} 1$ to $\mathrm{C} 3$ were the samples. The larger band was the internal control of PCR and it was the product of specific primers for the third intron of the DRB1 gene. The smaller sized band (186 bp) was the specific primer for the DQA1 * 05 allele.

Table 3. DRB1*0301-DQA1*0501 Frequency and Haplotype Associations with Type 1 Diabetes ${ }^{\text {a }}$

\begin{tabular}{|c|c|c|c|}
\hline Haplotypes & Frequency & OR & $95 \% \mathrm{CI}$ \\
\hline DRB1*0301'-DQA1*0501 & 0.01 & - & - \\
\hline $\mathrm{DRB1}^{*} 0301^{-}$-DQA1*0501 ${ }^{+}$ & 0.05 & 3.77 & $1.09 \pm 4.03$ \\
\hline DRB1*0301 $^{+}{ }^{+}$DQA1*0501 & 0.09 & 5.42 & $2.51 \pm 16.96$ \\
\hline $\mathrm{DRB1}^{*} 0301^{+}{ }^{-\mathrm{DQA1}}{ }^{*} 0501^{+}$ & 0.85 & 0.78 & $243.01 \pm 453$ \\
\hline
\end{tabular}

a Abbreviations: CI, Confidence interval; OR, Odds Ratio.

Table 4. DRB1*0301-DQA1*0501-DQB1*0201 Frequency and Haplotype Associations with Type 1 Diabetes ${ }^{\text {a }}$

\begin{tabular}{|c|c|c|c|}
\hline Haplotypes & Frequency & OR & $\mathbf{9 5} \% \mathrm{CI}$ \\
\hline $\begin{array}{l}\text { DRB1*0301'- DQA1*0501-- } \\
\text { DQB1*0201- }\end{array}$ & 0.04 & - & - \\
\hline $\begin{array}{l}\text { DRB1*0301'- DQA1*0501 }{ }^{+}- \\
\text {DQB1*0201- }\end{array}$ & 0.05 & 1.81 & $1.27 \pm 6.65$ \\
\hline $\begin{array}{l}\text { DRB1 }{ }^{*} 0301^{-}-\text {DQA1 }^{*} 0501^{-}- \\
\text {DQB1 }^{*} 0201^{+}\end{array}$ & 0.06 & 4.68 & $2.68 \pm 6.51$ \\
\hline $\begin{array}{l}\text { DRB1*0301 }{ }^{+} \text {- DQA1*0501-- } \\
\text { DQB1*0201- }^{*}{ }^{*}{ }^{-}\end{array}$ & 0.05 & 2.40 & $1.27 \pm 3$ \\
\hline $\begin{array}{l}\text { DRB1 }^{*} 0301^{+}{ }^{-} \text {DQA1*0501 } \\
\text { DQB1 }^{+} 0201^{+}\end{array}$ & 0.8 & 3.4 & $1.91 \pm 3.2$ \\
\hline
\end{tabular}

a Abbreviations: CI, Confidence interval; OR, Odds Ratio.

\section{Discussion}

Type 1 Diabetes is the consequence of progressive and selective destruction of pancreatic $\beta$ cells by an immunemediated process, leading to a lack of insulin. It has been well established that this self-destruction is primarily provoked by the activation of auto-reactive $\mathrm{T}$ lymphocytes by the production of T-helper 1 cytokines (24). In addition, the difference of the epidemiological data from one region to another could largely explain why the release of the autoimmunity is stimulated under the influence of one or more environmental factors, in genetically predisposed subjects. It is currently obvious that the strongest genetic susceptibility of predisposition is allotted to the IDDM1 alleles located in the HLA locus of the chromosome 6p21, and the non- HLA alleles, particularly the IDDM2 polymorph gene located in the region 5' of the insulin gene (INS) promoter situated on chromosome 11p15 (25). Previous studies showed that inheriting certain alleles of the HLA class II genes increases the probability of destruction of body's healthy cells by the immune cells (1).

Associations between HLA alleles and T1D began to be documented in many societies in the 1970s, first by serology and later by the use of molecular tools $(26,27)$. Common and unique DRB-DQB haplotypes conferring susceptibility to and protection against T1D were identified in each community; therefore, different HLA haplotypes have different effects on susceptibility to T1D, depending on the population under study (28-30). For example, certain HLA alleles, such as the DRB1*0301-DQB1*0201and DRB1*040101-DQB1*0302 haplotypes, increase the risk of T1D among Caucasians, while others like DRB1*1501DQB1*0602 appear to provide protection against T1D in all populations. The current study aimed for further characterization of the extended HLA haplotype DRB1*0301DQB1*0201-DQA1*0501, to identify the genetic susceptibility to T1D among the East Azerbaijan state population of Iran. Therefore, the frequencies of DRB1*0301, DQA1*0501, and DQB1*0201alleles and their haplotypes were analyzed in T1D patients and normal controls from this part of Iran to assess the contribution of HLA class II genes to T1D susceptibility. The findings of the current study showed that all three alleles are associated with T1D. The DRB1*0301 allele frequency distribution was higher among the patients. Based on the results mentioned above, it can be concluded that there is a highly significant association between these studied alleles and T1D. It can be construed that HLA-DR3-DQ2 haplotype has a very modest effect with respect to the risk of T1D. The information of this research study can also be useful as basic data for anthropology and disease association studies.

While considering the impact of the findings in the current study, important limitations must also be regarded. First, this study was a single-center case-controlled study, and subsequent investigations employing a larger number of patients and controls would be useful for confirm- 
ing the role of these alleles and their haplotypes in T1D risk. In other words, we are reaching the limits of statistical power, thus further work using larger sample sizes is required to unravel the T1D association in this region. Second, the association between the DR3-DQ2 haplotype and T1D was observed among subjects from the East Azerbaijan state of Iran. Finally, further studies are required to clarify this association among populations in different regions of Iran.

\section{Acknowledgements}

Many people have contributed to the development of this study and we would like to thank them all for their input. We specially thank the "immunology research center" of Tabriz University of Medical Sciences for their financial support. We also would like to thank the Ebne Sina medical genetic laboratory members for their help and guidance, and the participants for assisting in the collection of samples.

\section{Authors' Contributions}

We hereby submit a manuscript entitled "Evaluation of the association between Human Leukocyte Antigen class II DR3-DQ2 haplotype and type 1 diabetes in children of the East Azerbaijan state of Iran" by Fatemeh Zeinali Sehrig, Mahmoud Shekari Khaniani, Nasrin Sohrabi, Siamak Shiva, Behzad Baradan and Sima Mansoori Derakhshan, to be considered for publication as an original article. Fatemeh Zeinali Sehrig and Sima Mansoori Derakhshan designed the study, performed the experiments and wrote the article. Nasrin Sohrabi participated in the sample collection. Siamak Shiva, Mahmoud Shekari Khaniani and Behzad Baradan contributed to the analysis and interpretation of data.

\section{Funding/Support}

The "immunology research center" of Tabriz university of medical sciences provided financial support.

\section{References}

1. Buc M, Bucova M, Javor J, Krivosikova M, Stuchlikova M, Shawkatova I, et al. Associations between HLA class II alleles and type 1 diabetes mellitus in the Slovak population. Endocr Regul. 2006;40(1):1-6.

2. Nejentsev S, Howson JM, Walker NM, Szeszko J, Field SF, Stevens $\mathrm{HE}$, et al. Localization of type 1 diabetes susceptibility to the MHC class I genes HLA-B and HLA-A. Nature. 2007;450(7171):887-92.

3. Stayoussef M, Benmansour J, Al-Irhayim AQ, Said HB, Rayana CB, Mahjoub T, et al. Autoimmune type 1 diabetes genetic susceptibility encoded by human leukocyte antigen DRB1 and DQB1 genes in Tunisia. Clin Vaccine Immunol. 2009;16(8):1146-50.

4. Lie BA, Todd JA, Pociot F, Nerup J, Akselsen HE, Joner G, et al. The predisposition to type 1 diabetes linked to the human leukocyte antigen complex includes at least one non-class II gene. Am J Hum Genet.1999;64(3):793-800.

5. Karamifar H, Habibian N, Amirhakimi G, Karamizadeh Z, Alipour A. Adiponectin is a Good Marker for Metabolic State among Type 1 Diabetes Mellitus Patients. Iran J Pediatr. 2013;23(3):295-301.

6. Dorman J. Molecular epidemiology of insulin-dependent diabetes mellitus: WHO DiaMond Project. WHO DiaMond Molecular Epidemiology Sub-Project Group. Gac Med Mex. 1997;133 Suppl 1:151-4.

7. Dorman JS, LaPorte RE, Stone RA, Trucco M. Worldwide differences in the incidence of type I diabetes are associated with amino acid variation at position 57 of the HLA-DQ beta chain. Proc Natl Acad Sci U S A. 1990;87(19):7370-4.

8. Bartsocas CS, Gerasimidi-Vazeou A. Genetics of type 1 diabetes mellitus. Pediatr Endocrinol Rev. 2006;3 Suppl 3:508-13.

9. Stayoussef M, Benmansour J, Al-Jenaidi FA, Nemr R, Ali ME, Mahjoub $\mathrm{T}$, et al. Influence of common and specific HLA-DRB1/DQB1 haplotypes on genetic susceptibilities of three distinct Arab populations to type 1 diabetes. Clin Vaccine Immunol. 2009;16(1):136-8.

10. Erlich H, Valdes AM, Noble J, Carlson JA, Varney M, Concannon P, et al. HLA DR-DQ haplotypes and genotypes and type 1 diabetes risk: analysis of the type 1 diabetes genetics consortium families. Diabetes. 2008;57(4):1084-92.

11. Florez JC, Hirschhorn J, Altshuler D. The inherited basis of diabetes mellitus: implications for the genetic analysis of complex traits. Annu Rev Genomics Hum Genet. 2003;4:257-91.

12. American Diabetes A. Diagnosis and classification of diabetes mellitus. Diabetes Care. 2008;31 Suppl 1:S55-60.

13. Barrett JC, Clayton DG, Concannon P, Akolkar B, Cooper JD, Erlich $\mathrm{HA}$, et al. Genome-wide association study and meta-analysis find that over 40 loci affect risk of type 1 diabetes. Nat Genet. 2009;41(6):703-7.

14. Cooper JD, Smyth DJ, Smiles AM, Plagnol V, Walker NM, Allen JE, et al. Meta-analysis of genome-wide association study data identifies additional type 1 diabetes risk loci. Nat Genet. 2008;40(12):1399-401.

15. Cerna M, Novota P, Kolostova K, Cejkova P, Zdarsky E, Novakova D, et al. HLA in Czech adult patients with autoimmune diabetes mellitus: comparison with Czech children with type 1 diabetes and patients with type 2 diabetes. Eur J Immunogenet. 2003;30(6):401-7.

16. Herr M, Dudbridge F, Zavattari P, Cucca F, Guja C, March R, et al. Evaluation of fine mapping strategies for a multifactorial disease locus: systematic linkage and association analysis of IDDM1 in the HLA region on chromosome 6p21. Hum Mol Genet. 2000;9(9):1291-301.

17. Marsh SG, Albert ED, Bodmer WF, Bontrop RE, Dupont B, Erlich HA, et al. Nomenclature for factors of the HLA system, 2002. Eur J Immunogenet. 2002;29(6):463-515.

18. Robinson J, Waller MJ, Parham P, de Groot N, Bontrop R, Kennedy LJ, et al. IMGT/HLA and IMGT/MHC: sequence databases for the study of the major histocompatibility complex. Nucleic Acids Res. 2003;31(1):311-4.

19. She JX. Susceptibility to type I diabetes: HLA-DQ and DR revisited. Immunol Today.1996;17(7):323-9.

20. Undlien DE, Lie BA, Thorsby E. HLA complex genes in type 1 diabetes and other autoimmune diseases. Which genes are involved? Trends Genet. 2001;17(2):93-100.

21. Almawi WY, Busson M, Tamim H, Al-Harbi EM, Finan RR, WakimGhorayeb SF, et al. HLA class II profile and distribution of HLADRB1 and HLA-DQB1 alleles and haplotypes among Lebanese and Bahraini Arabs. Clin Diagn Lab Immunol. 2004;11(4):770-4.

22. Sacks DB, Bruns DE, Goldstein DE, Maclaren NK, McDonald JM, Parrott M. Guidelines and Recommendations for Laboratory Analysis in the Diagnosis and Management of Diabetes Mellitus. Clin Chem. 2002;48(3):436-72.

23. Olerup O, Zetterquist H. HLA-DR typing by PCR amplification with sequence-specific primers (PCR-SSP) in 2 hours: an alternative to serological DR typing in clinical practice including donorrecipient matching in cadaveric transplantation. Tissue Antigens. 1992;39(5):225-35.

24. Kulmala P, Savola K, Petersen JS, Vahasalo P, Karjalainen J, Lopponen T, et al. Prediction of insulin-dependent diabetes mellitus in siblings of children with diabetes. A population-based study. The Childhood Diabetes in Finland Study Group. J Clin Invest. 1998;101(2):327-36.

25. Todd JA, Acha-Orbea H, Bell JI, Chao N, Fronek Z, Jacob CO, et al. A molecular basis for MHC class II--associated autoimmunity. Sci- 


\section{Mansoori Derakhshan S et al.}

ence. 1988;240(4855):1003-9.

26. Nejentsev S, Gombos Z, Laine AP, Veijola R, Knip M, Simell O, et al. Non-class II HLA gene associated with type 1 diabetes maps to the 240-kb region near HLA-B. Diabetes. 2000;49(12):2217-21.

27. Zavattari P, Lampis R, Motzo C, Loddo M, Mulargia A, Whalen M, et al. Conditional linkage disequilibrium analysis of a complex disease superlocus, IDDM1 in the HLA region, reveals the presence of independent modifying gene effects influencing the type 1 diabetes risk encoded by the major HLA-DQB1, -DRB1 disease loci. Hum Mol Genet. 2001;10(8):881-9.

28. Ionescu-Tirgoviste C, Guja C, Herr M, Cucca E, Welsh K, Bunce M, et al. Low frequency of HLA DRB1*03 - DQB1*02 and DQB1*0302 haplotypes in Romania is consistent with the country's low in cidence of Type I diabetes. Diabetologia. 2001;44 Suppl 3:B60-6.

29. Al-Jenaidi FA, Wakim-Ghorayeb SF, Al-Abbasi A, Arekat MR, IraniHakime N, Najm P, et al. Contribution of selective HLA-DRB1/ DQB1 alleles and haplotypes to the genetic susceptibility of type 1 diabetes among Lebanese and Bahraini Arabs. J Clin Endocrinol Metab. 2005;90(9):5104-9.

30. Noorian S, Sayarifard F, Farhadi E, Barbetti F, Rezaei N. GCK Mutation in a Child with Maturity Onset Diabetes of the Young, Type 2. Iran J Pediatr. 2013;23(2):226-8. 